\title{
EFFECTS OF ACTUATED PELVIS CONTROL ON SHEAR AND PRESSURE DISTRIBUTION
}

\author{
P. van Geffen ${ }^{1}$, H.F.J.M. Koopman ${ }^{1}$, J. Reenalda ${ }^{2}$ and P.H. Veltink ${ }^{3}$ \\ ${ }^{1}$ Laboratory of Biomechanical Engineering, University of Twente, Enschede, The Netherlands \\ ${ }^{2}$ Roessingh Research and Development, Rehabilitation Centre ' $t$ Roessingh, Enschede, The Netherlands \\ ${ }^{3}$ Biomedical Signals and Systems, University of Twente, Enschede, The Netherlands \\ Email: P.vanGeffen@ctw.utwente.nl, web1:www.bw.ctw.utwente.nl,web2: www.dynasit.nl
}

\section{INTRODUCTION}

Among wheelchair dependent patients, a poor sitting posture is often seen [1] which contributes to all kinds of physical problems during long term sitting [2]. Because pelvic tilt is crucial for the adopted sitting posture [3], chair configurations enforcing proper pelvic alignment are necessary. Common methods to individualise chair configurations are either based on postural stability [4], pressure distribution, lumbar load [2] or minimizing shear forces [5]. To define postural criteria and to optimize chair configuration, most studies involved experimental analyses based on seating forces and pressure distribution. The objective of the present study is to propose a concept for independent pelvis control and to analyse the effects on shear force and seating pressure.

\section{METHODS}

In figure $1 \mathrm{~A}$, a schematic representation is shown of an adopted sitting posture imposed by the configuration of a 'normal' chair. Figure 1B shows a concept in which a connecting bar linked the seat and back support. Aligning the pelvis with the connecting bar makes independent pelvic control possible. Figure 1C shows a mechanical concept for independent body segment actuation.
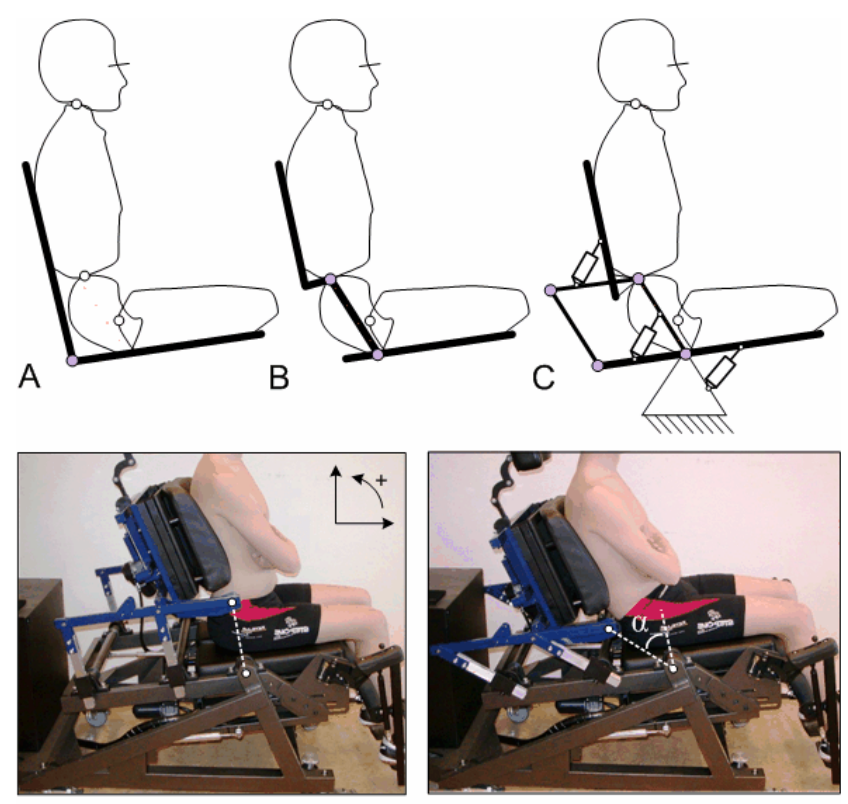

D

E

Figure 1

Experiments were done with an instrumented chair containing the concept for independent body segment control. Chair configurations enforcing anterior and posterior pelvic tilt are shown in figures 1D and 1E. Six uni-axial force sensors were mounded in the seat frame to measure seating forces. A Tekscan pressure mapping system was used to measure pressure distribution. A dispersion index (DI) determined tuberal load by dividing total seating pressure by the pressure under the tuberosities. High indexes indicate high tuberal load. From a specific chair configuration (seat and back support were tilted 10 and 25 degrees) pelvic tilt $(\alpha)$ was actuated relative to the seat within a range of 50 degrees $\left(0^{\circ}-50^{\circ}\right)$. The experiment involved measurements in which one healthy subject participated. Five cycles of actuated pelvic movements were recorded, averaged, normalized (\% max) and related to DI and shear force (shear).

\section{RESULTS AND DISCUSSION}

Figure 2 show pelvic tilt, DI and shear during one cycle of pelvic movement. Great influence was found on both shear and DI. Excessive pelvic anterior tilt resulted to increase the amount of shear and to decrease DI. Pelvic anterior tilt was limited by passive stiffness of the hip joint. Prolonged anterior tilt causes shear forces to increase and the tuberosities to unload. Since the increase in shear force was not exerted on the tuberosities, no decubitus risk was expected. Furthermore, 'neutral' pelvis angle $\left(\alpha^{*}\right)$ involving minimal shear forces was derived and shown in figure 2. It was suggested that the observed shear difference in this position was caused by visco-elastic tissue properties around the hip joint.

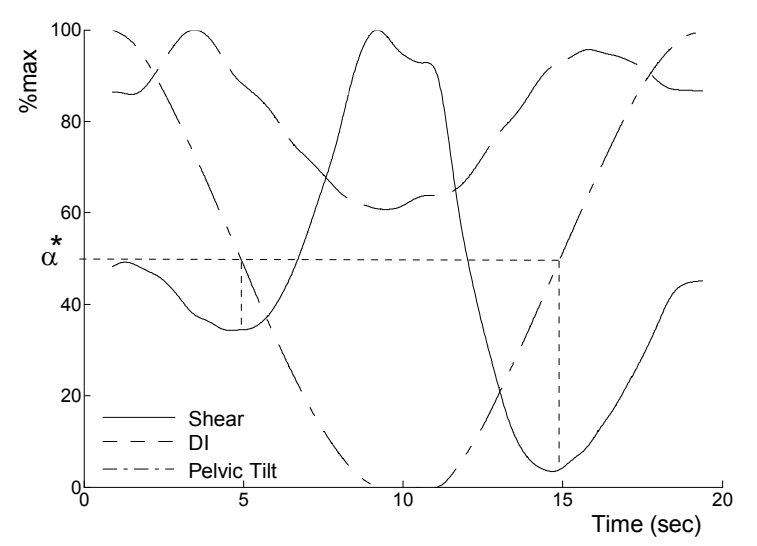

Figure 2

\section{CONCLUSION}

The proposed concept for independent pelvis control seems useful to individualize chair configuration involving proper pelvic alignment and decubitus prevention.

\section{REFERCENCES}

1. M. Alm, et al., (2003), Spinal Cord, 41, (10), 563-71

2. M. Makhsous, et al., (2003), Spine, 28, (11), 1113-21; discussion 1121-2

3. S. Sprigle, et al., (2002), Arch Phys Med Rehabil, 83, (11), 1597-602

4. Y. J. Janssen-Potten, et al., (2001), Arch Phys Med Rehabil, 82, (10), 1393-402

5. R. H. Goossens, et al., (1995), J Biomech, 28, (2), 22530 